American Journal of Infectious Diseases 3 (4): 202-207, 2007

ISSN 1553-6203

(C) 2007 Science Publications

\title{
Immunodeficient Parameters in the HIV-1 Transgenic Rat Model
}

\author{
${ }^{1}$ Sulie L. Chang, ${ }^{2}$ Frank Ocasio and ${ }^{1}$ Jose A.Beltran \\ ${ }^{1}$ Department of Biological Sciences, Seton Hall University, South Orange, NJ 07079 \\ ${ }^{2}$ New York Presbyterian Hospital, Weill Cornell Medical Center, New York, NY 10021
}

\begin{abstract}
Recently an HIV-1 transgenic (HIV-1Tg) rat model was created that carries a gag-poldeleted HIV-1 genome under the control of the HIV-1 viral promoter. However, other viral proteins are expressed in most organs and tissues, and are found in the circulating blood. Since HIV-1 targets the immune system in humans, we examined two immunological parameters, leukocyte-endothelial adhesion (LEA) and inflammatory cytokine production, in 5 mo old HIV-1Tg rats to identify immune functions that may be impaired even before the onset of symptoms of HIV-1 infection. We administered a single injection (i.p.) of the bacterial endotoxin, lipopolysaccharide (LPS, $250 \mathrm{ug} / \mathrm{kg}$ ), to 5 mo old HIV-1Tg rats, age-matched transgenic control (Tg) rats, and F344/NHsd (F344) control background strain rats. LPS induced an LEA response in both the Tg control and F344 control animals. However, in the HIV-1Tg rats, there was no LEA response to LPS. Following LPS administration, there was significantly greater serum levels of TNF- $\alpha$ and IL- $1 \beta$, two pro-inflammatory cytokines, in the HIV-1Tg rats compared to the control animals. In contrast, the serum level of IL-10, an anti-inflammatory cytokine, was comparable in the HIV-1Tg, Tg control, and F344 control rats. Our data show that, in the HIV-1Tg rat, there is a negative correlation between the LEA response and the induction of pro-inflammatory cytokines in response to bacterial endotoxin. These findings suggest that the persistent presence of viral proteins may be, at least, partially responsible for the immunodeficiency that occurs with HIV-1 infection, and that the HIV-1Tg rat could be a valid rodent model in which to study various aspects of HIV-1 infection.
\end{abstract}

Key Words: Leukocyte-endothelial adhesion, cytokines, lipopolysaccharide

\section{INTRODUCTION}

Human immunodeficiency virus-1 (HIV-1) infection results from the actions of HIV-1 viral proteins, including the envelope glycoprotein 120 (gp120) and Tat, on targeted cells of the immune system, such as macrophages and T-lymphocytes ${ }^{[1,6] \text {. }}$ HIV-1 infection is marked by an array of pathologies and a variety of secondary infections ${ }^{[7-11]}$, and can progress to full-blown AIDS. In AIDS, the lethal outcome from opportunistic infections results from impairment of various immune functions ${ }^{[8,9,12,14]}$. Highly active anti-retroviral therapy (HAART) consists of inhibitors that target viral entry, reverse transcriptase and viral protease to control viral replication, restore immunity, and delay disease progression, but it cannot eliminate the viral infection ${ }^{[13,15,18]}$. The clinical challenge in this post-HAART era is, therefore, the persistent viral infection that results from the presence of HIV viral proteins in the host ${ }^{[8,9,18] \text {. }}$
The HIV-1 transgenic (HIV-1Tg) rat model was developed at the University of Maryland Biotechnology Institute (UMBI) from F344/NHsd and Hsd SpraugeDawley ${ }^{\circledR} \mathrm{SD}^{\circledR}$ rat stock ${ }^{[19],}$ and is currently available at Harlan, Inc. [Indianapolis, IN]. The HIV-1Tg rat carries a gag-pol-deleted HIV-1 genome under the control of the HIV-1 viral promoter, and expresses 7 of the 9 HIV genes ${ }^{[19] .}$ Thus, while there is no viral replication in the HIV-1Tg rat model, viral proteins are expressed in various organs ${ }^{[19]}$, and are found in the

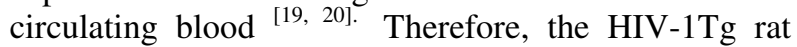
seems to mimic the condition of patients given HAART, who have limited (controlled) viral replication, but persistent HIV infection.

HIV-1Tg rats exhibit both differences and similarities when compared to the age-matched F344 control animals. The HIV-1Tg rats are lower in body weight in comparison to age matched controls. However, they show no signs of retarded growth, and they progressively gain weight similar to the control animals. Even though the HIV-1Tg rats eat and drink

Corresponding Author: Sulie L. Chang, Department of Biological Sciences, Seton Hall University, 400 South Orange Avenue, South Orange, NJ 07079; Tel: 973-761-9456, Fax: 973-275-2489 
less than the F344 control rats, there is no evidence of anorexia. There are also no signs of anhedonia in the HIV-1Tg animals, based on their taste response to sucrose [unpublished data]. The HIV-1Tg rats demonstrate poorer performance in a water maze, based on auditory, olfactory, and textual cues, and they appear to have some learning deficits even before they develop symptomatic signs of HIV infection [unpublished data].

While HIV-1 is primarily an infection of the immune system in humans, many immune functions have not yet been characterized in the new HIV-1Tg rat model.We, therefore, examined specific immunological functions in the HIV-1Tg rat to identify immune parameters that may be impaired even before the development of symptoms of HIV infection.

During normal physiological conditions, leukocytes travel along the vessel wall, particularly in venules, at a constant velocity, intermittently coming in contact with the endothelium [21, 22]. During inflammatory conditions, these leukocytes adhere to the endothelium of the venules ${ }^{[21]}$ in order to penetrate the endothelium, enter the underlying tissue, and travel to the site of infection. This leukocyte-endothelial adhesion (LEA) is the prelude to many cellular immune events, including migration toward a chemotactic stimulus, phagocytosis, intracellular degradation, and cytokine release. LEA is also the initial step by which leukocytes in the peripheral blood cross the vascular endothelial cell lining of vital organs, including the brain and heart ${ }^{[23,26] .}$ Thus, LEA is one of the body's first lines of defense against invading pathogens ${ }^{[21]}$.

LEA is a complex physiological process mediated by various adhesion molecules found on both leukocytes and the vascular endothelium ${ }^{[26]}$. It can be induced by several factors, including the bacterial endotoxin such as lipopolysaccharide (LPS), and the bacterial chemotactic factor, N-formyl-methionylleucyl-phenylalanine (FMLP). LPS-induced LEA occurs due to increased expression of cell adhesion molecules on the vascular endothelium, and LPS' effects are long-lasting (hours or days) ${ }^{[28] .}$ In contrast, FMLP induces LEA by increasing adhesion molecules on the surface of the leukocytes, and its effects are transient (minutes) ${ }^{[28] . ~ T h u s, ~ t o p i c a l ~ s u f f u s i o n ~ w i t h ~}$ FMLP at a low concentration $\left(10^{-7} \mathrm{M}\right)$ is routinely used in intravital microscopy to confirm that the leukocytes are capable of responding to a stimulus and forming the LEA response.

LPS is routinely used as a model molecule to induce inflammation in vivo. In addition to inducing LEA, LPS can initiate an inflammatory response by stimulating the release of cytokines, including TNFalpha (TNF- $\alpha)$, interleukin-1beta (IL-1 $\beta)$, and interleukin-10 (IL-10). Both TNF- $\alpha$ and IL-1 $\beta$ are proinflammatory cytokines. They are mainly secreted by macrophages and are important mediators of inflammatory responses such as LEA. They are also involved in a variety of cellular activities, including cell proliferation, differentiation, and apoptosis ${ }^{[29-31]}$. On the other hand, IL-10 is an anti-inflammatory cytokine. It is produced mainly by macrophages and has pleiotropic effects in regulating the inflammation ${ }^{[32,33] \text {. }}$

In this study, we examined the LEA response to systemic treatment with LPS in the HIV-1Tg rats. Intravital microscopy was used to directly measure LEA in the mesentery venules of the HIV-1Tg rats. We also compared the serum levels of two proinflammatory cytokines, TNF- $\alpha$ and IL- $1 \beta$, and one anti-inflammatory cytokine, IL-10, using the enzymelinked immunosorbant assay (ELISA). Even though the serum levels of all the cytokines examined were greater in the HIV-1Tg rats compared to the control animals, the HIV-1Tg rats failed to exert an LEA response following treatment with LPS.

\section{MATERIALS AND METHODS}

Materials: Lipopolysaccharide (LPS) from Salmonella typhimurium and n-formyl-methionyl-leucylphenylalanine (FMLP) were obtained from Sigma Chemical Co. (St. Louis, Mo). Sodium pentobarbital was obtained from Veterinary Laboratories, Inc. (Lenexa, KS).

Animals: Male HIV-1Tg rats, transgenic control (Tg) animals, and F344/NHsd (F344) control background strain rats were purchased from Harlan, Inc. (Indianapolis, IN). One to three animals were housed in standard plastic rodent cages or in flexible-film isolators in a temperature-controlled environment with standard rat diet and water available ad libitum. The animals used in these studies were 5 mo of age. All experimental procedures were conducted during the light period of a 12-h light/dark cycle, and were approved by the Institutional Animal Care and Use Committee of Seton Hall University.

Treatment with lipopolysaccharide: Six HIV-1Tg rats, $6 \mathrm{Tg}$ control rats, and $6 \mathrm{~F} 344$ rats (all 5 mo of age) were randomly assigned to receive an injection with either LPS $(250 \mu \mathrm{g} / \mathrm{kg}$, i.p. $)$ or an equal volume of saline. Two hours after the injection, about $1.0 \mathrm{~mL}$ of blood was collected from the tail vein of each rat and used to prepare serum

Intravital microscopy: At the end of the treatment with LPS described above, the rats were anesthetized with sodium pentobarbital ( $45 \mathrm{mg} / \mathrm{kg}$, i.p.) and prepared 
Am. J. Infect. Dis., 3 (4): 202-207, 2007

for intravital microscopy as described in Occasio et al.,

[34]. Pentobarbital has been shown to have no influence on leukocyte endothelial adhesion (LEA) [35]. A tracheotomy was performed to facilitate the animal's respiration, and the carotid artery was cannulated in order to monitor the blood pressure during the intravital microscopy. The animal was placed on a heating pad; its mesentery was exteriorized through a mid-sagittal abdominal incision and draped over a transparent glass shelf. The tissues were topically suffused with a $1 \%$ Ringer's lactate-gelatin drip solution maintained at $37^{\circ}$ C. The intestines were covered with moist gauze, and the areas outside the region of interest were covered with Saran wrap. The mesentery was observed with a Nikon UM3 metallurgic microscope adapted for intravital microscopy. A Nikon $10 \mathrm{X}$ water immersion lens was used to view the field on a Panasonic TR930A high-resolution video monitor. LEA in the mesenteric venules was recorded using a Panasonic VWCD-52 video camera and a JVC HR-D630U videocassette recorder. Leukocytes sticking to the vessel endothelium for more than $30 \mathrm{sec}$ were counted as adhering leukocytes, and expressed as the number of adhering cells per $100 \mu \mathrm{m}$ of vessel length.

During the course of intravital microscopy, inflammation in the mesenteric vessels was acutely and transiently induced with a 2 min topical suffusion with the chemoattractant, FMLP $\left(10^{-7} \mathrm{M}\right)$. Stock FMLP was made fresh by dissolving the powder in DMSO (Fisher Scientific, Fair Lawn, NJ). The stock solution of FMLP was then dissolved in the isotonic bicarbonate Ringer's lactate-gelatin drip solution, $\mathrm{pH}$ 7.4. DMSO at the concentration present in the solution $\left(1.4 \times 10^{-7} \mathrm{M}\right)$ has been shown to have no effect on leukocyte-endothelial interactions ${ }^{[21] \text {. }}$

Enzyme-linked immunosorbant assay (ELISA) for

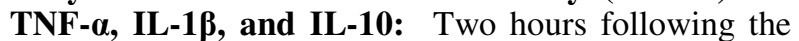
injection with LPS $(250 \mu \mathrm{g} / \mathrm{kg}$, i.p. $)$, tail vein blood was collected from each animal and allowed to clot for at least $30 \mathrm{~min}$. The blood samples were used to prepare serum by centrifugation at $4^{\circ} \mathrm{C}(1000 \mathrm{x} g)$ for $30 \mathrm{~min}$. The sera were stored in aliquots at $-80^{\circ} \mathrm{C}$ until use in the ELISA. ELISA was performed according to the manufacturer's protocol (R \& D Systems, Minneapolis, $\mathrm{MN})$.

Statistical analysis: The LEA data are presented as the mean \pm SE. Comparisons of data were made by a oneway analysis of variance (ANOVA) followed by a Newman-Keuls post-hoc test, or by a paired or unpaired Student's t-test (Mann-Whitney). Significance was assessed at the $95 \%(\mathrm{P}<0.05)$ confidence level. Significance was defined as a probability of 0.05 or less.

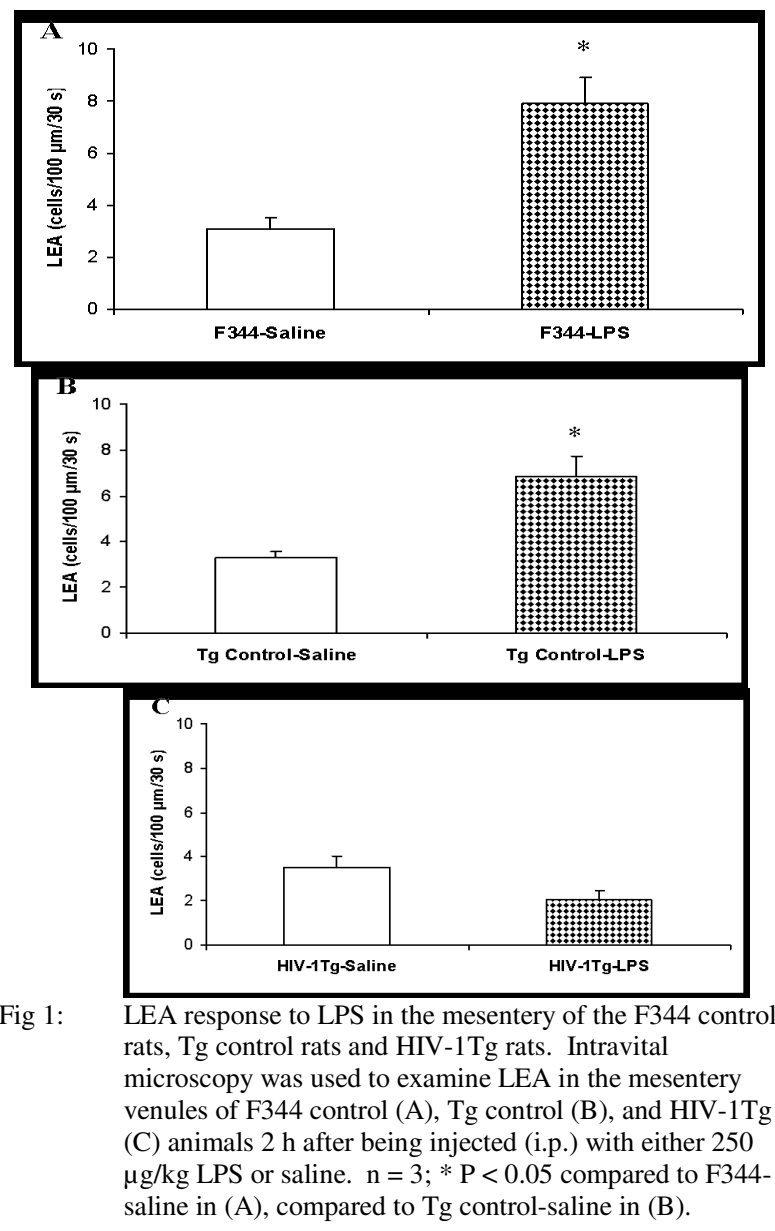

\section{RESULTS}

The LPS-induced LEA response in the HIV-1 Tg rats: Leukocyte-endothelial adhesion (LEA) is the initial step in the inflammatory response cascade which allows leukocytes to cross the vascular endothelial cell barrier to go to the site of tissue injury. Administration of an endotoxin, such as lipopolysaccharide (LPS), via intraperitoneal (i.p.) injection has been used as a model to induce LEA in vivo. Topical suffusion with the chemoattractant, FMLP $\left(10^{-7} \mathrm{M}\right)$, was used to confirm that all the animals had functional LEA response. As shown in Fig. 1, in response to an injection with LPS, only the LEA in the F344 control (A) and Tg control (B) rats was significantly elevated compared to the animals treated given saline $(\mathrm{P}<0.05)$. However, LEA was not elevated in the HIV-1Tg rats $(\mathrm{C})$ in response to LPS compared to saline treated animals. FMLP 
Am. J. Infect. Dis., 3 (4): 202-207, 2007

Table 1: Topical suffusion with FMLP $\left(10^{-7} \mathrm{M}\right)$ induction of LEA (cells/100 $\mu \mathrm{m} / 30 \mathrm{~s}$ ) in F344 control, Tg control and HIV-1Tg rats given with and without LPS $(250 \mu \mathrm{g} / \mathrm{kg})$

\begin{tabular}{lllll}
\hline & Saline & Saline-FMLP & Saline-LPS & Saline-LPS-FMLP \\
\hline F344 Control & $3.1 \pm 0.45$ & $11.4 \pm 1.5 *$ & $7.9 \pm 0.9$ & $22.3 \pm 1.4 *$ \\
Tg Control & $3.3 \pm 0.29$ & $12.1 \pm 1.1 *$ & $6.9 \pm 0.9$ & $13.1 \pm 1.1^{*}$ \\
HIV-1Tg & $3.5 \pm 0.52$ & $12.4 \pm 0.8 *$ & $2.1 \pm 0.4$ & $4.3 \pm 0.5 *$ \\
\hline
\end{tabular}

Table 2: Serum levels of IL-1 $\beta$, TNF- $\alpha$, and IL-10 in F344, Tg control, and HIV-1Tg rats as determined by ELISA.

\begin{tabular}{|c|c|c|c|c|c|c|}
\hline & \multicolumn{2}{|c|}{ F344 rats } & \multicolumn{2}{|c|}{ Tg rats } & \multicolumn{2}{|c|}{ HIV-1Tg rats } \\
\hline & Saline & LPS & Saline & LPS & Saline & LPS \\
\hline $\mathrm{IL}-1 \beta(\mathrm{pg} / \mathrm{mL})$ & $80.5 \pm 1.4$ & $214.7 \pm 2.6^{*}$ & $98.3 \pm 3.0$ & $253.2 \pm 5.7^{*}$ & $204.8 \pm 3.3$ & $1392.0 \pm 9.1$ *\#户 \\
\hline $\mathrm{TNF}-\alpha(\mathrm{pg} / \mathrm{mL})$ & $129.7 \pm 5.7$ & $183.7 \pm 3.5^{*}$ & $129.5 \pm 5.4$ & $203.3 \pm 5.6^{*}$ & $112.7 \pm 7.5$ & $4557.1 \pm 145.9^{\text {*\#t }}$ \\
\hline IL-10 (pg/mL) & $127.4 \pm 4.5$ & $188.0 \pm 2.1^{*}$ & $125.6 \pm 3.4$ & $214.1 \pm 2.7^{*}$ & $136.4 \pm 2.8$ & $358.8 \pm 9.8^{\text {*\# }}$ \\
\hline
\end{tabular}

$\mathrm{N}=3$ for each group.

* $\mathrm{P}<0.05$ compared to animals without suffusion with FMLP; \# $\mathrm{P}<0.05$ compared to F344 control rats treated with LPS; $\dagger \mathrm{P}<0.05$ compared to $\mathrm{Tg}$ control rats treated with LPS.

suffusion confirmed that all six groups of animals, F344 control rats with and without LPS, Tg control rats with and without LPS, and HIV-1Tg rats with and without LPS (Table 1),could exert a transient LEA response to the chemoattractrant.

LPS-induced serum levels of cytokines in the HIV1Tg rats: The actions of LPS are thought to be mediated via the autocrine actions of the cytokines secreted in response to LPS ${ }^{[36,37]}$. As shown in Table 2, LPS increased the serum levels of TNF- $\alpha$ and IL-1 $\beta$ by about 1-1.5-fold in the F344 rats. The LPS-induced increase in IL-1 $\beta$ and TNF- $\alpha$ was 7 -fold and 38 -fold higher, respectively, in the HIV Tg rats compared to the control F344 animals $(\mathrm{P}<0.05)$. There was a 0.8 -fold increase in IL-10 in response to LPS in the F344 rats (Table 2), and a 1.5-fold increase in IL-10 in the HIV Tg rats $(\mathrm{P}<0.05)$.

\section{DISCUSSION}

Infection with the HIV-1 virus results in a large variety of pathologies ${ }^{[7-11]}$, and can progress to fullblown AIDS. Patients with AIDS generally succumb to opportunistic infections due to impairment of various immune functions and responses ${ }^{[8,9,12-14]}$. In this study, we examined two immunological parameters, the LEA response and the production of cytokines involved with the inflammatory response, in the new HIV-1Tg rat model.

The induction of LEA in the microcirculation is one of the first immune responses to infection $\left.{ }^{[38}\right]$, and can be induced by several agents, including the chemotactic factor, FMLP, and the bacterial endotoxin, LPS, ${ }^{[21,34,39] .}$ All six groups of animals tested (F344, $\mathrm{Tg}$ control, and HIV-1Tg rats treated with either LPS or saline) produced a similar LEA response to topical suffusion with FMLP, indicating that the adhesion molecules expressed on the leukocytes of all of the animals should be adequate to adhere to the vascular endothelium. In both the F344 and Tg control rats, the LEA response to LPS occurred as expected. In contrast, in the HIV-1Tg animals, LPS failed to induce an LEA response. Two possible reasons for the lack of an LPS-induced LEA response in the HIV-1Tg rats are: (1) There may be inadequate expression of adhesion molecules on the endothelial cells; and, (2) the microcirculation environment may be different in the presence of HIV-1 viral proteins.

LPS can also induce the production of cytokines, such as TNF- $\alpha$, IL- $1 \beta$, and IL10, which are involved in the inflammatory response to infection. The serum levels of the two pro-inflammatory cytokines, TNF- $\alpha$ and IL- $1 \beta$, was significantly greater in the HIV- $1 \mathrm{Tg}$ rats than in the two control animals in response to LPS, whereas the level of the anti-inflammatory cytokine, IL10 , in response to LPS in the HIV-1Tg rats was comparable to that in both the F344 and Tg control animals. Thus, it appears that there is an imbalance of pro- versus anti-inflammatory cytokines induced by LPS in the HIV-1Tg rats, which could have detrimental effects on other immune responses, such as LEA.

Interestingly, in the HIV-1Tg rats, the production of the two pro-inflammatory cytokines, TNF- $\alpha$ and IL$1 \beta$, following LPS administration did not correlate with the induction of the LEA response as it did in both 
control animal groups. The over-production of proinflammatory cytokines and failure of the LEA response in the HIV-1Tg rats following a single treatment with a non-pyrogenic dose of LPS, as shown in this study, suggests that the persistent presence of the HIV-1 viral proteins may result in dysregulation of immune function, and in particular, with the failure of the LEA response to an immune challenge. It may be that an inadequate LEA response is one of the immune impairments by which patients with HIV-1 infection succumb to opportunistic bacterial infections. Further studies are needed to determine the molecular and cellular mechanisms underlying the failure of the LEA response to LPS in the HIV-1Tg rats.

In summary, HIV-1Tg rats are immunodeficient in many respects, even when they do not show signs of illness. The persistent presence of HIV viral proteins in these animals may be sufficient to result in the impairment of immune responses, such as LEA, and an imbalance in the production of inflammatory cytokines in response to subsequent bacterial infection. Thus, the HIV-1Tg rat could be a valid rodent model in which to investigate various physiological functions and immune responses to bacterial agents in the presence of HIV-1 infection.

\section{ACKNOWLEDGEMENT}

This work was supported, in part, by R01 grants, DA 007058, K02 DA 016149, and R21 DA019836 to SLC.

\section{REFERENCES}

1. Clap ham, P. R., and A. McKnight, 2001. HIV-1 receptors and cell tropism. Br. Med. Bull., 58: 43-59.

2. Cicala, C., J. Arthos, S. M. Selig, G. Dennis, Jr., D. A. Hosack, D. Van Ryk, M. L. Spangler, T. D. Steenbeke, P. Khazanie, N. Gupta, J. Yang, M. Daucher, R. A. Lempicki, and A. S. Fauci, 2002. HIV envelope induces a cascade of cell signals in non-proliferating target cells that favor virus replication. Proc. Natl. Acad. Sci. U. S. A., 99: 9380-9385.

3. Jiang, J., and C. Aiken, 2006. Maturation of the viral core enhances the fusion of HIV-1 particles with primary human $\mathrm{T}$ cells and monocyte-derived macrophages. Virology., 346: 460-468.

4. Torensma, R., and C. G. Figdor, 2002. The Achilles' heel of HIV. Med. Hypotheses., 58: 386387.

5. Trkola, A, 2004. HIV-host interactions: vital to the virus and key to its inhibition. Curr. Opin. Microbiol., 7: 555-559.
6. Verani, A., G. Gras, and G. Pancino, 2005. Macrophages and HIV-1: dangerous liaisons. Mol. Immunol., 42: 195-212.

7. Popovic, M., and S. Gartner, 1989. Biology of human imunodeficiency virus: virus receptor and cell tropism. Curr. Opin. Immunol., 1: 516-520.

8. Jones, L. E., and A. S. Perelson, 2005. Opportunistic infection as a cause of transient viremia in chronically infected HIV patients under treatment with HAART. Bull. Math. Biol., 67: 1227-1251.

9. Lucas, S, 2001. Update on the pathology of AIDS. Intensive Crit. Care Nurs., 17: 155-166.

10. Miles, K, 2005. Primary HIV infection. Community Pract., 78: 331-333.

11. Rumbaugh, J. A., and A. Nath, 2006. Developments in HIV neuropathogenesis. Curr. Pharm. Des., 12: 1023-1044.

12. Sierra, S., B. Kupfer, and R. Kaiser, 2005. Basics of the virology of HIV-1 and its replication. J. Clin. Virol., 34: 233-244.

13. Sleasman, J. W., and M. M. Goodenow, 2003. 13. HIV-1 infection. J. Allergy Clin. Immunol., 111: S582-592.

14. Nagappan, V., and P. Kazanjian, 2005. Bacterial infections in adult HIV-infected patients. HIV Clin. Trials, 6: 213-228.

15. Gianotti, N., and A. Lazzarin, 2005. Sequencing antiretroviral drugs for long-lasting suppression of HIV replication. New Microbiol., 28: 281-297.

16. Agbottah, E., N. Zhang, S. Dadgar, A. Pumfery, J. D. Wade, C. Zeng, and F. Kashanchi, 2006. Inhibition of HIV-1 virus replication using small soluble Tat peptides. Virology, 345: 373-389.

17. Barbaro, G., A. Scozzafava, A. Mastrolorenzo, and C. T. Supuran, 2005. Highly active antiretroviral therapy: current state of the art, new agents and their pharmacological interactions useful for improving therapeutic outcome. Curr. Pharm. Des., 11: 1805-1843.

18. Vigano, A., D. Trabattoni, L. Schneider, F. Ottaviani, A. Aliffi, E. Longhi, S. Rusconi, and M. Clerici, 2006. Failure to eradicate HIV despite fully successful HAART initiated in the first days of life. J. Pediatr., 148: 389-391.

19. Reid, W., M. Sadowska, F. Denaro, S. Rao, J. Foulke, Jr., ill, R. Kamin-Lewis, C. Wei, P. Ray, R. C. Gallo, M. Reitz, and J. Bryant, 2001. An HIV-1 transgenic rat that develops HIV-related pathology and immunologic dysfunction. Proc. Natl. Acad. Sci. U. S. A., 98: 9271-9276.

20. Mazzucchelli, R., M. Amadio, S. Curreli, F. Denaro, K. Bemis, W. Reid, J. Bryant, A. Riva, M. Galli, and D. Zella, 2004. Establishment of an ex vivo model of monocytes-derived macrophages differentiated from peripheral blood mononuclear cells (PBMCs) from HIV-1 transgenic rats. Mol. Immunol., 41: 979-984. 
21. House, S. D., and H. H. Lipowsky, 1987. Leukocyte-endothelium adhesion: microhemodynamics in mesentery of the cat. Microvasc. Res., 34: 363-379.

22. Nazziola, E., and S. D. House, 1992. Effects of hydrodynamics and leukocyte-endothelium specificity on leukocyte-endothelium interactions. Microvasc. Res., 44: 127-142.

23. Callera, G. E., A. C. Montezano, R. M. Touyz, T. M. Zorn, M. H. Carvalho, Z. B. Fortes, D. Nigro, E. L. Schiffrin, and R. C. Tostes, 2004. ETA receptor mediates altered leukocyte-endothelial cell interaction and adhesion molecules expression in DOCA-salt rats. Hypertension, 43: 872-879.

24. Ishikawa, M., E. Sekizuka, C. Oshio, S. Sato, N. Yamaguchi, S. Terao, K. Tsukada, H. Minamitani, and T. Kawase, 2002. Platelet adhesion and arteriolar dilation in the photothrombosis: observation with the rat closed cranial and spinal windows. J. Neurol. Sci., 194: 59-69.

25. Jones, S. P., S. D. Trocha, M. B. Strange, D. N. Granger, C. G. Kevil, D. C. Bullard, and D. J. Lefer, 2000. Leukocyte and endothelial cell adhesion molecules in a chronic murine model of myocardial reperfusion injury. Am. J. Physiol. Heart Circ. Physiol., 279: H2196-2201.

26. Villringer, A., U. Dirnagl, A. Them, L. Schurer, F. Krombach, and K. M. Einhaupl, 1991. Imaging of leukocytes within the rat brain cortex in vivo. Microvasc. Res., 42: 305-315.

27. Smith, C. W, 1993. Endothelial adhesion molecules and their role in inflammation. Can. J. Physiol. Pharmacol., 71: 76-87.

28. Tonnesen, M. G., D. C. Anderson, T. A. Springer, A. Knedler, N. Avdi, and P. M. Henson, 1989. Adherence of neutrophils to cultured human microvascular endothelial cells. Stimulation by chemotactic peptides and lipid mediators and dependence upon the Mac-1, LFA-1, p150,95 glycoprotein family. J. Clin. Invest. 83: 637-646.

29. Aggarwal, B. B, 2003. Signalling pathways of the TNF superfamily: a double-edged sword. Nat. Rev. Immunol., 3: 745-756.

30. Gosselin, D., and S. Rivest, 2007. Role of IL-1 and TNF in the brain: Twenty years of progress on a Dr. Jekyll/Mr. Hyde duality of the innate immune system. Brain Behav. Immun., 21: 281-289.
31. Tseng, J., J. Do, J. H. Widdicombe, and T. E. Machen, 2006. Innate immune responses of human tracheal epithelium to Pseudomonas aeruginosa flagellin, TNF-alpha, and IL-1beta. Am. J. Physiol. Cell Physiol., 290: C678-690.

32. Moore, K. W., R. de Waal Malefyt, R. L. Coffman, and A. O'Garra, 2001. Interleukin-10 and the interleukin-10 receptor. Annu. Rev. Immunol. 19: 683-765.

33. Yoshikai, Y, 2006. [Crosstalk between innate and adaptive immunity]. Nippon Rinsho., 64: 12231228.

34. Ocasio, F. M., Y. Jiang, S. D. House, and S. L. Chang, 2004. Chronic morphine accelerates the progression of lipopolysaccharide-induced sepsis to septic shock. J. Neuroimmunol., 149: 90-100.

35. Janssen, G. H., G. J. Tangelder, M. G. oude Egbrink, and R. S. Reneman, 1997. Different effects of anesthetics on spontaneous leukocyte rolling in rat skin. Int. J. Microcirc. Clin. Exp., 17: 305-313.

36. Asami, T., T. Ito, H. Fukumitsu, H. Nomoto, Y. Furukawa, and S. Furukawa, 2006. Autocrine activation of cultured macrophages by brainderived neurotrophic factor. Biochem. Biophys. Res. Commun., 344: 941-947.

37. Cassatella, M. A., L. Meda, S. Bonora, M. Ceska, and G. Constantin, 1993. Interleukin 10 (IL-10) inhibits the release of proinflammatory cytokines from human polymorphonuclear leukocytes. Evidence for an autocrine role of tumor necrosis factor and IL-1 beta in mediating the production of IL-8 triggered by lipopolysaccharide. J. Exp. Med., 178: 2207-2211

38. Asimakopoulos, G., and K. M. Taylor, 1998. Effects of cardiopulmonary bypass on leukocyte and endothelial adhesion molecules. Ann. Thorac. Surg., 66: 2135-2144.

39. House, S. D., X. Mao, G. Wu, D. Espinelli, W. X. Li, and S. L. Chang, 2001. Chronic morphine potentiates the inflammatory response by disrupting interleukin-1beta modulation of the hypothalamic-pituitary-adrenal axis. J. Neuroimmunol., 118: 277-285. 